\title{
ADVANCEMENTS IN ESTIMATING CROP GROWTH STAGES USING RADARSAT-2 AND TERRASAR-X POLARIMETRIC DATA
}

\author{
George Lampropoulos*, Yifeng Li, Ting Liu \\ A.U.G. Signals Ltd., Toronto, Canada, \\ lampro@augsignals.com; yifeng@augsignals.com; tliu@augsignals.com
}

THEME: Synergetic Use C/X

KEY WORDS: Polarimetric SAR, RADARSAT-2, TerraSAR-X, crop growth stages monitoring, polarimetric decomposition, polarimetric parameters integration

\begin{abstract}
:
This paper uses RADARSAT-2 quad Polarimetric Synthetic Aperture Radar (PolSAR) and TerraSAR-X dual polarimetric SAR data to monitor agriculture crop growth stages. Two RADARSAT-2 Fine Quad Wide (FQW) beam modes FQ2W and FQ10W, each with 5 sets of data and 13 sets of Stripmap TerraSAR-X data were used in the study. Both RADARSAT-2 POLSAR data and TerraSARX data were acquired in summer 2012 outside Winnipeg, Manitoba, Canada. The study was carried out to two crop types: canola and wheat, each contains 5 regions of interest from ground truth crop classification map in the image scene. Polarimetric features such as differential reflectivity bands ratio, entropy, anisotropy, alpha angle, lambda, scattering diversity and polarization index were evaluated for two crop types. The results from both RADARSAT-2 and TerraSAR-X data were compared and they demonstrated clear relationships between crop growth stages and polarimetric parameters. It is observed that entropy, lambda and differential reflectivity from both data have similar responses to crop growth stages in their common coverage period. The results were also validated using ground truth information.
\end{abstract}

\section{INTRUDUCTION}

Using multiple satellites such as RADARSAT-2 and TerraSAR$\mathrm{X}$ POLSAR data to accurately estimate crop growth stages is an important agriculture application. This can enable growers to proactively prevent disease, increase yields, and enhance longterm planning. Current agricultural practices rely heavily on time-consuming site visits, which can delay responses to potential threats and limit the extent of data that can be acquired for large tracts of land. There is high potential to maximize the use of satellite data to acquire comprehensive crop information quickly, automatically, and with high accuracy, to better manage large areas. Moreover, as many crops are susceptible to diseases and infestations during the narrow windows of specific growth stages, (e.g., canola's vulnerable flowering period, which lasts only 14 to 21 days on average), the estimation of crop growth stages, phenology and location is vital in order to identify which fields are at risk and when.

Currently, PolSAR data utilization for crop management applications has limited researches on crop growth stage estimation [Cloutis2010, McNairn2004, McNairn2014]. Agriculture and Agri-Food Canada and other organizations have conducted preliminary research in this area using RADARSAT-2 and TerraSAR-X data to examine crop conditions in the Soil Moisture Active Passive mission Validation Experiment 2012 (SMAPVEX12) campaign in summer 2012, Manitoba, Canada. The preliminary study in this paper is to demonstrate that by incorporating multi-sensor information, such as RADARSAT-2 and TERRASAR-X data, and considering many more polarimetric parameters, accurate crop stage classification and identification can be achieved.

\section{METHODOLOGY}

At different crop growth stages, crop characteristics will change. In general, the agriculture industry defines ten distinct stages of crop growth [Earth2012]. PolSAR sensors will capture the vegetation difference in $\mathrm{C}$ - and $\mathrm{X}$ - bands. Various polarization parameters will be calculated for both RADARSAT- 2 and TerraSAR-X data in this paper to find out the their responses to the crop stage changing. The purpose of this work is to find possible polarimetric parameters of crop growth stages for different crops. The technology provided in this work will provide possible estimation for different crop growth stages.

The RADARSAT-2 fully Polarimetric SAR (PolSAR) system measures for each resolution element in the scene, producing a scattering matrix:

$$
[S]=\left[\begin{array}{ll}
S_{H H} & S_{H V} \\
S_{V H} & S_{V V}
\end{array}\right]
$$

The scattering matrix elements in Pauli basis can be arranged into a measurement vector, which is called the scattering vector

$$
\vec{k}=\frac{1}{\sqrt{2}}\left[S_{H H}+S_{V V}, S_{H H}-S_{V V}, 2 S_{V H}\right]^{T}
$$

The equation is under the reciprocal assumption, with the three elements referred to as the Pauli components of the signal.

Various polarimetric parameters can be obtained based on these three complex elements $S_{H H}, S_{V V}$ and $S_{H V}$ :

- Differential Reflectivity Ratio of scattering matrices

\footnotetext{
* Corresponding author.
} 
For quad-polarized data, the three differential reflectivity ratios are $\frac{S_{H H}-S_{V V}}{S_{V V}}, \frac{S_{H H}-S_{H V}}{S_{H V}}$ and $\frac{S_{H V}-S_{V V}}{S_{V V}}$.

- Polarimetric entropy, anisotropy, $\alpha$ angle and lambda $\lambda$ As proposed by Cloude and Pottier [Cloude1996], the average coherency matrix

$$
\langle T\rangle=\left\langle\vec{k} \vec{k}^{+}\right\rangle
$$

can be decomposed by means of eigen decomposition as

$$
\langle T\rangle=\lambda_{1} k_{1} \vec{k}_{1}^{+}+\lambda_{2} k_{2} \vec{k}_{2}^{+}+\lambda_{3} k_{3} \vec{k}_{3}^{+}
$$

and is interpreted as an incoherent sum of three fully deterministic scattering mechanisms, described by eigenvectors $\vec{k}_{i}$ and weighted by eigen values $\lambda_{i}$.

The polarimetric entropy is defined by

$$
H=\sum_{i=1}^{3}-\frac{\lambda_{i}}{\sum_{j=1}^{3} \lambda_{j}} \ln \left(\frac{\lambda_{i}}{\sum_{j=1}^{3} \lambda_{j}}\right), 0 \leq H \leq 1
$$

Polarimetric entropy represents the randomness of the scattering. Zero entropy indicates a single scattering mechanism and unity entropy indicates a random mixture of scattering mechanisms, i.e. a depolarizing target. Values in between indicate the degree of dominance of one particular scatterer.

The polarimetric anisotropy, given by

$$
A=\frac{\lambda_{2}-\lambda_{3}}{\lambda_{2}+\lambda_{3}}, 0 \leq A \leq 1
$$

is a ratio of eigen values and its higher value indicates multiple scatterers.

The $\alpha$ angle is calculated based upon the eigenvectors and is a number indicative of the average or dominant scattering mechanism:

$$
\alpha=\frac{\lambda_{1}}{\sum_{j=1}^{3} \lambda_{j}} \alpha_{1}+\frac{\lambda_{2}}{\sum_{j=1}^{3} \lambda_{j}} \alpha_{2}+\frac{\lambda_{3}}{\sum_{j=1}^{3} \lambda_{j}} \alpha_{3}
$$

where $\alpha_{i}$ can be calculated by describing the eigenvectors $\vec{k}_{i}$. In complex spherical coordinates [Cloude1996]. The lower limit of $0^{\circ}$ indicates surface scattering, $45^{\circ}$ indicates dipole or volume scattering, while the upper limit of $90^{\circ}$ represents a dihedral reflector or multiple scattering.

The lambda, $\lambda$, is the mean magnitude of the mechanism, and can be obtained as

$$
\lambda=\frac{\lambda_{1}^{2}+\lambda_{2}^{2}+\lambda_{3}^{2}}{\sum_{j=1}^{3} \lambda_{j}}
$$

\section{- $\quad$ Scattering diversity}

The scattering diversity is defined as [Praks09]

$$
\hat{H}=\frac{3}{2}\left(1-\|N\|_{F}^{2}\right)
$$

where

$$
N=\left\langle\vec{k} \vec{k}^{+}\right\rangle^{-1}\left\langle\vec{k} \vec{k}^{+}\right\rangle=\frac{T}{\operatorname{trace}(T)}
$$

is called the power-normalized coherency matrix, and $\|*\|_{F}$ is the Frobenius norm [Lu96]. $\hat{H}$ and $H$ are highly correlated.

\section{- Depolarization index}

The depolarization index is defined as the Euclidian distance of the normalized Mueller matrix to the ideal depolarizer of the nonreciprocal case [Praks09]. D(M) can be expressed in terms of elements of the coherency matrix as follows:

$$
d(M)=\frac{\sqrt{\sum_{i, j} T_{i j}^{2}-\frac{(\operatorname{trace}(T))^{2}}{4}}}{\frac{\operatorname{trace}(T)}{2}}
$$

which can be expressed here as twice the Euclidian distance of the normalized coherency matrix $\mathrm{N}$ to the ideal depolarizer in the nonreciprocal case.

All polarimetric parameters will be calculated using both RADARSAT-2 and TerraSAR-X polarimetric data. The evaluation and comparison studies will also be carried out for crop growth stage estimation.

\section{RADARSAT-2 AND TERRASAR-X DATA}

The dataset used in this paper was acquired from the Soil Moisture Active Passive mission Validation Experiment 2012 (SMAPVEX12) campaign [SMAPVEX2012a], in summer 2012, Manitoba, Canada.

The RADARSAT- 2 and TerraSAR-X data used in this paper is listed in Table 1. Two RADARSAT-2 Wide Fine Quad Polarization beam modes of FQ2W and FQ10W were used. FQ2W data has incidence angles from $19.0^{\circ}$ to $22.7^{\circ}$, while FQ10W has incidence angles from $28.4^{\circ}$ to $31.6^{\circ}$. Both beam modes are in descending orbit. TerraSAR-X data was in Stripmap StripNear-005 beam mode, and are also in descending orbit. TerraSAR-X data has duel polarization with $\mathrm{HH}$ and $\mathrm{VV}$ polarizations and incidence angles from $26.0^{\circ}$ to $26.7^{\circ}$. The RADARSAT-2 data covers shorter crop growth period from April to July, each beam mode has 24-day date interval between two acquisitions. By utilizing both FQ2W and FQ10W data, a 10-day and 14-day date intervals were generated as shown in Table 1 for more frequent coverage of the crop growth season. The TerraSAR-X data has 11-day date interval and covers longer crop growth period from May to September.

The different crops are identified based on the ground truth information provided by SMAPVEX12 crop classification map file [SMAPVEX12b]. Canola and wheat crop types each has 5 different regions of interest which were used in estimating the polarimetric parameters of RADARSAT- 2 and TerraSAR-X data as shown in Figures 1 and 2.

\section{PRELIMINARY RESULTS AND DISCUSSIONS}

Different crop types are staged differently with times. Two crops are considered in this paper, canola and wheat, since there are sufficient fields on the ground for evaluation their polarimetric parameters based on the ground truth crop classification map.

The data listed in Table 1 has been georeferenced/orthorectified in order to overlay with ground truth vector data. Figures 1 and 2 illustrate RADARSAT-2 and 
TerrsSAR-X images where the crop ground truth regions of interest are overlaid.

\begin{tabular}{|c|c|c|c|c|c|}
\hline \multicolumn{4}{|c|}{ RADARSAT-2 } & \multicolumn{2}{|c|}{ TerraSAR-X } \\
\hline $\begin{array}{l}\text { Date } \\
\text { index }\end{array}$ & FQ2W & $\begin{array}{l}\text { Date } \\
\text { index }\end{array}$ & FQ10W & $\begin{array}{l}\text { Date } \\
\text { index }\end{array}$ & $\begin{array}{l}\text { StripNear- } \\
005 \mathrm{R}\end{array}$ \\
\hline 1 & April16 & 2 & April26 & 1 & May 08 \\
\hline 3 & May10 & 4 & May20 & 2 & May 19 \\
\hline 5 & June03 & 6 & June 13 & 3 & May 30 \\
\hline 7 & June27 & 8 & July 07 & 4 & June 10 \\
\hline 9 & July 21 & 10 & July 31 & 5 & June 21 \\
\hline & & & & 6 & July 02 \\
\hline & & & & 7 & July 13 \\
\hline & & & & 8 & July 24 \\
\hline & & & & 9 & August 04 \\
\hline & & & & 10 & August 15 \\
\hline & & & & 11 & August 26 \\
\hline & & & & 12 & $\begin{array}{l}\text { September } \\
06\end{array}$ \\
\hline & & & & 13 & $\begin{array}{l}\text { September } \\
17\end{array}$ \\
\hline
\end{tabular}

Table 1. RADARSAT-2 and TerraSAR-X Data

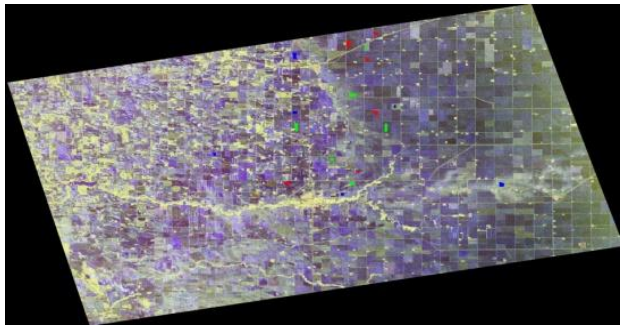

Figure 1. Pauli Image of RADARSAT-2 acquired in April 16, 2012 in FQ2W mode, where crop regions of interest are overlaid. Red: Canola; Green: Corn; Blue: Wheat

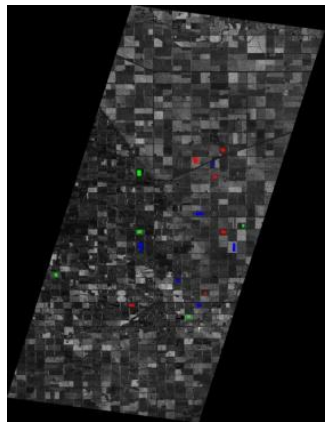

Figure 2. HH Image of TerraSAR-X acquired in May 30, 2012 in StipNear-005mode, where crop regions of interest are overlaid. Red: Canola; Green: Corn; Blue: Wheat

The speckle reduction has also been performed to reduce noises inherited in the SAR image. A Polarimetric Gaussian-BoxCar Speckle filtering was used on a Coherency matrix T3 which performs incoherent averaging within a 5 by 5 sliding window. Figure 3 is an example of speckle reduced TerraSAR-X HH image subset. It can be seen that speckle reduced image is less random and the contrast is significantly enhanced.

Different polarimetric parameters are calculated using speckle reduced images for both RADARSAT-2 and TerraSAR-X images for two different crop types, canola and wheat.
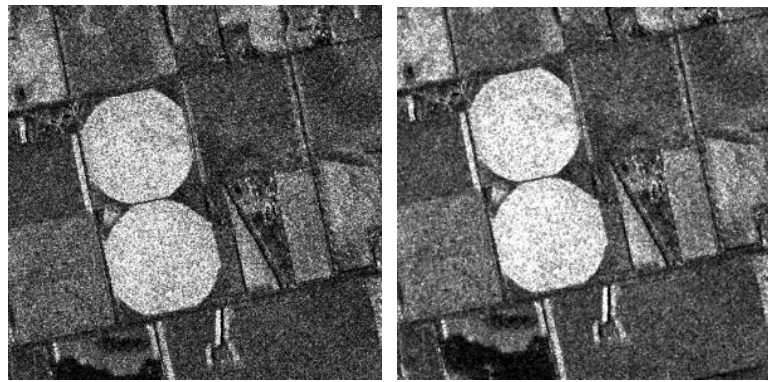

Figure 3. A speckle reduced images using Gaussian BoxCar filter for polarimetric TerraSAR-X HH polarization, window site $5 \times 5$

\subsection{Canola crop type}

Canola covered the largest area at 1,295 and 2,828 thousand hectares in Manitoba 2009. It plays significant role in agriculture planning and management. Selected polarimetric parameters of canola crop type of both RADARSAT-2 and TerraSAR-X are depicted in Figure 4 and 5 for comparison.

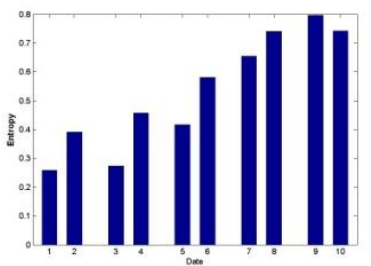

a) RADARSAT-2 entropy

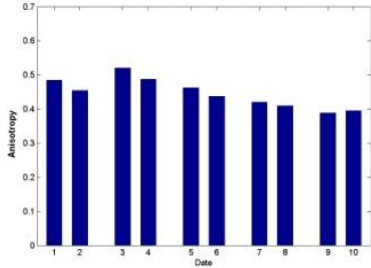

c) RADARSAT-2 anisotropy

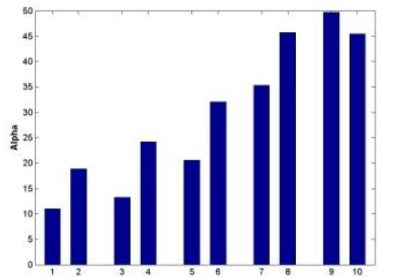

e) RADARSAT-2 alpha angle

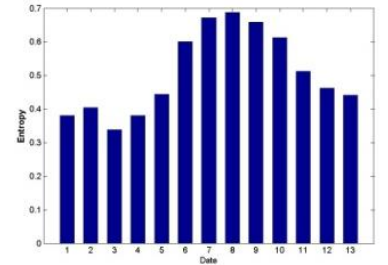

b) TerraSAR-X entropy

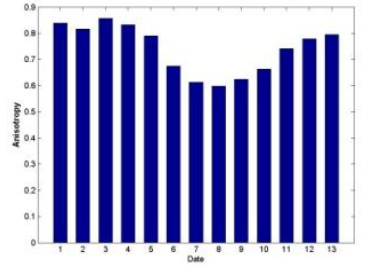

d) TerraSAR-X anisotropy

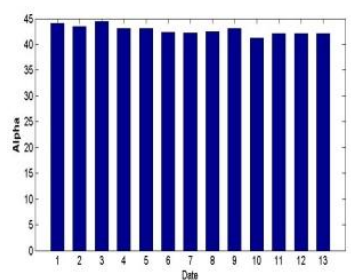

f) TerraSAR-X alpha angle 


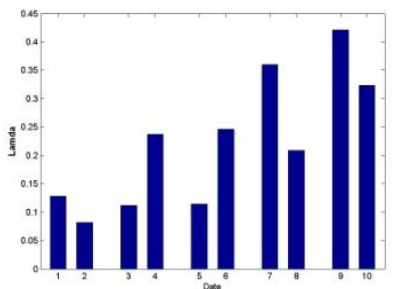

g) RADARSAT-2 Lambda

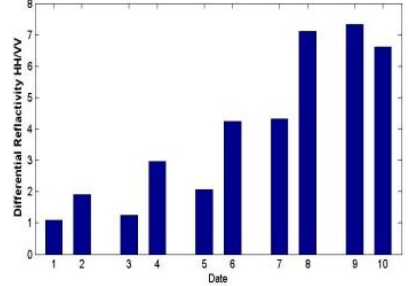

i) RADARSAT-2 differential reflectivity of $\mathrm{HH} / \mathrm{VV}$

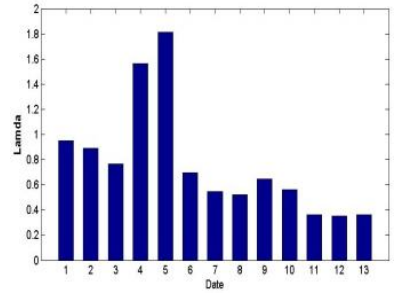

h) TerraSAR-X Lambda

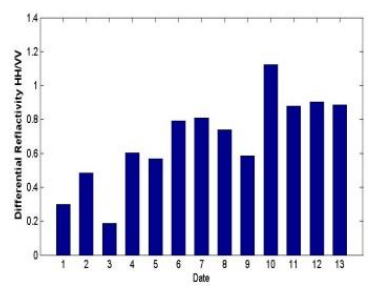

j) TerraSAR-X differential reflectivity of $\mathrm{HH} / \mathrm{VV}$
Figure 4. RADARSAT-2 and TerraSAR-X polarimetric parameter comparison for canola crop type. Date in $\mathrm{X}$-axis is from April 16 to July 31 for RADARSAT-2, and May 8 to

September 17 for TerraSAR-X as shown in Table 1

The X-axis in all figures refers to the date index in Table 1 of RADARSAT- 2 and TerraSAR-X data. The RADARSAT- 2 date index 3-10 and TerraSAR-X date index 1-8 cover approximately the same crop growth time from May to end of July.

In addition to the common polarimetric parameters for both RADARSAT-2 and TerraSAR-X displayed in Figure 4, more RADARSAT-2 polarimetric features are displayed in Figure 5, which also demonstrate the changes with crop growth stages.

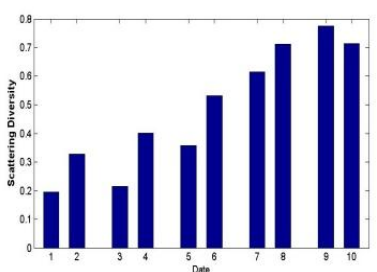

a) RADARSAT-2 Scattering diversity

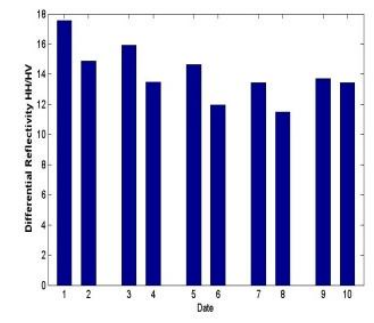

c) RADARSAT-2

Differential Reflectivity of $\mathrm{HH} / \mathrm{HV}$

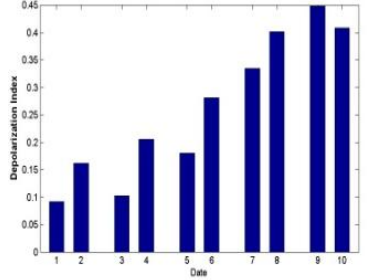

b) RADARSAT-2 Depolarization index

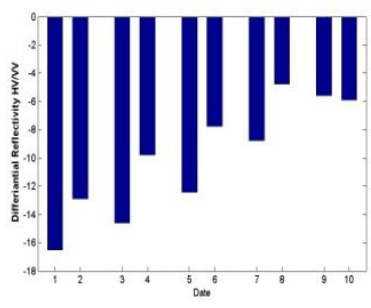

d) RADARSAT-2

Differential Reflectivity of $\mathrm{HV} / \mathrm{VV}$
Figure 5. Additional RADARSAT-2 polarimetric parameters of canola crop type. Date in X-axis is from April 16 to July 31 for RADARSAT- 2 as shown in Table 1

\subsection{Wheat crop type}

In Manitoba wheat is planted between May 1st and May 31st and is harvested between August and October. In 2009 and 2010 wheat covered the second largest seeded area of all crops at 1, 228 and 4,000 thousand hectares. Polarimetric features of both RADARSAT-2 and TerraSAR-X data for wheat crop type are depicted in Figure 6 for comparison.

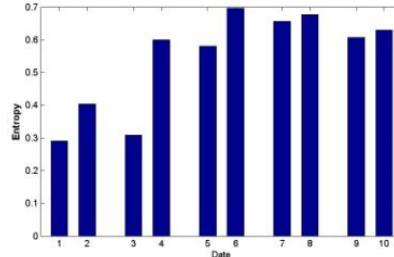

a) RADARSAT-2 entropy

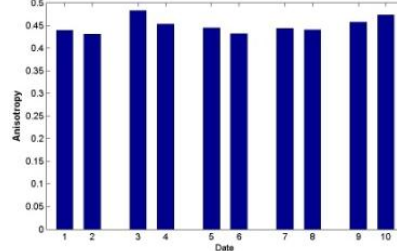

c) RADARSAT-2 Anisotropy

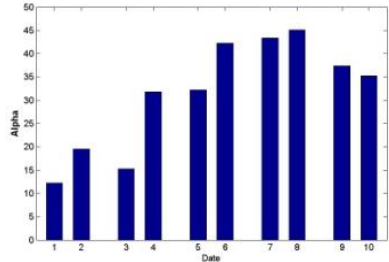

e)RADARSAT-2 alpha angle

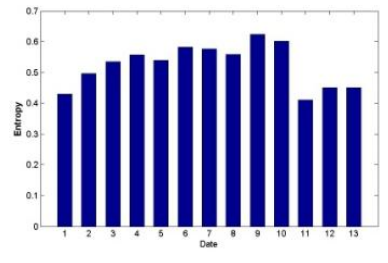

b) TerraSAR-X entropy

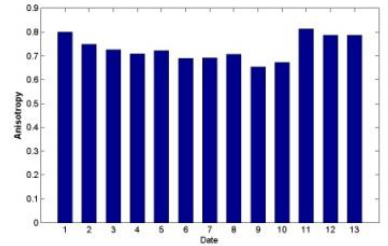

d)TerraSAR-X anisotropy

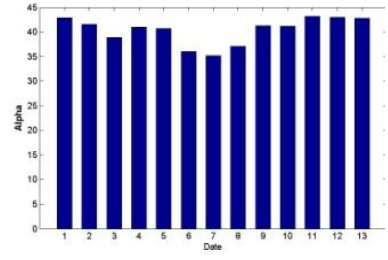

f) TerraSAR-X alpha angle

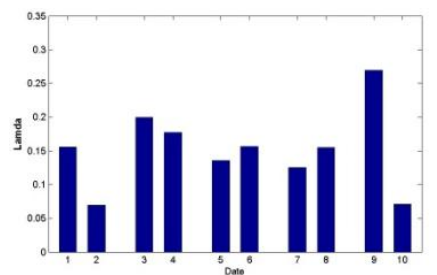

g) RADARSAT-2 Lambda

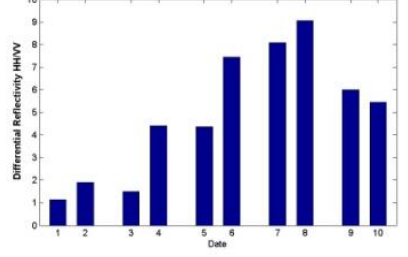

i) RADARSAT-2 differential reflectivity of $\mathrm{HH} / \mathrm{VV}$

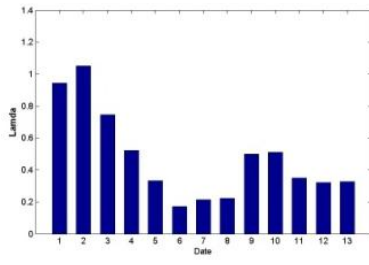

h) TerraSAR-X Lambda

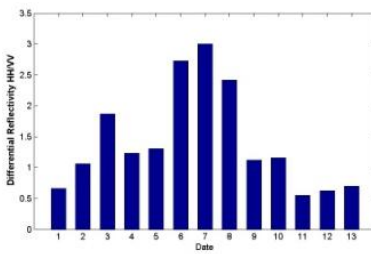

j) TerraSAR- $X$ differential reflectivity of $\mathrm{HH} / \mathrm{VV}$
Figure 6. RADARSAT-2 and TerraSAR-X polarimetric parameter comparison for wheat crop type. Date in X-axis is from April 16 to July 31 for RADARSAT-2, and May 8 to September 17 for TerraSAR-X as Shown in Table 1

Similar as in canola crop type case, additional RADARSAT-2 polarimetric parameters are also given in Figure 7. 


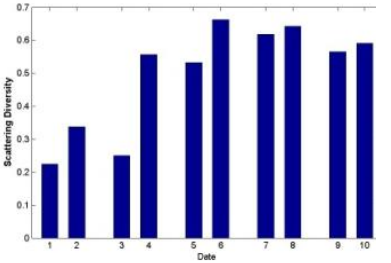

a) RADARSAT-2Scattering diversity

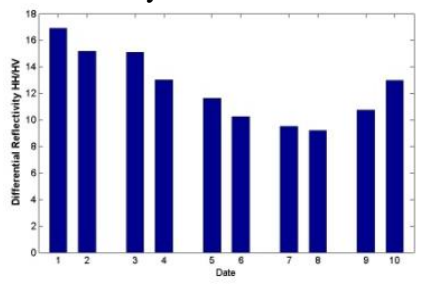

c) RADARSAT-2 Differential Reflectivity of $\mathrm{HH} / \mathrm{HV}$

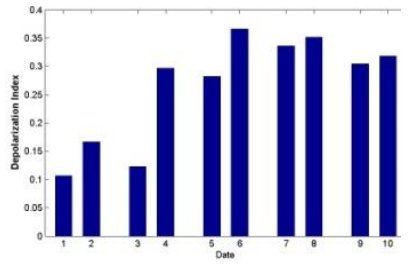

b) RADARSAT-2 Depolarization index

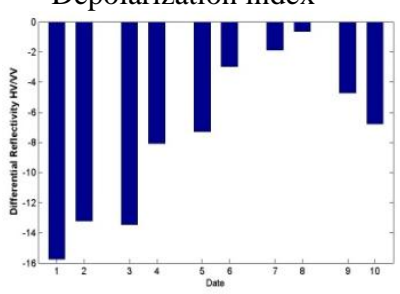

d) RADARSAT-2 Differential Reflectivity of $\mathrm{HV} / \mathrm{VV}$
Figure 7. Additional RADARSAT-2 polarimetric parameters of wheat crop type. Date in X-axis is from April 16 to July 31 for RADARSAT-2 as shown in Table 1

\subsection{Discussions}

The preliminary results indicate that some POLSAR polarimetric parameters may be sensitive enough to changes in crop structure to be of value in determining phenology. When crops growth stages are in germinations and leave development, SAR backscatter is dominated by surface/soil scattering. As crop emerges and canopies develop, SAR response primarily originates from multiple scattering events reflected from the leaves, stalks and fruit of the canopy and the soil. As the crop transits to seed development stage, leaf area declines, but biomass increases. A thick canopy creates significant multiple scattering and increases cross-polarization backscatter [McNairn2014]. For all the stages, some PolSAR polarimetric parameters will change with vegetation volume of crop structure.

As observed from the preliminary results, two beam modes of FQ2W and FQ10W of RADARSAT-2 parametric parameters have similar trend of changes although they have different incidence angles. The fluctuation of the results may be due to various factors such as rainfall, soil moisture and temperature. Furthermore, both the X-Band and C-Band entropy have very similar fashion of responses in their common growth covering period for both canola and wheat (TerraSAR-X date 1-8). RADARSAT-2 alpha angle changes more significantly with growth stage than that of the TerraSAR-X data. It is expected that alpha angle changes from surface scattering to volume scattering when crop grows. RADARSAT-2 alpha angle reflected the crop growth stage while TerraSAR-X alpha angle is keeping close to $45^{\circ}$ indicating volume scattering dominates during the growth stages due to its dual polarization status and smaller X-Band wavelength. The differential reflectivity of $\mathrm{HH} / \mathrm{VV}$ for both C- and X- bands data are high at end of July for canola and low after July for TerraSAR-X data. This is when canola flowers begin to emerge from buds. It is also observed that the differential reflectivity for wheat reaches high value at end of June and early July for both RADARSAT-2 and TerraSAR-X data followed by a significant drop. Other polarimetric parameters of RADARSAT-2 also indicate significant changes with crop growth stages for both canola and wheat.

\section{CONCLUSIONS}

This paper examined both TerraSAR-X and RADARSAT-2 polarimetric SAR data to determine if these sensors could be useful in estimating crop growth stages. The preliminary results indicate that some SAR polarimetric parameters are responsive to crop growth stages for canola and wheat. Those polarimetric parameters include entropy, alpha angle, lambda and differential reflectivity of HH/VV for both RADARSAT2 and TerraSAR-X. Due to shorter repeat time of the TerraSAR-X data, more growth covering period provides more complete crop growth stages. On the other hand, RADARSAT-2 Quad polarization provides more polarimetric parameters which demonstrate strong correlations between those parameters and crop growth stages.

Additional data which covers more growth stages with precise ground truth information are required for future work. The combination/integration of both RADARSAT-2 and TerraSAR-X results are also expected to enhance the performance and accuracy of estimating of the crop growth stages. It is expected that the combined and utilized both Cand $\mathrm{X}$ - bands data will provide the final indicators for better estimation of the different crop growth stages.

\section{ACKNOWLEDGEMENTS}

This on-going research is partially funded by Canadian Space Agency and supported by DLR for TerraSAR-X data.

\section{REFERENCES}

[Cloutis2010] E.A.Cloutis, "Agricultural crop monitoring using ariborne multi-spectral imagery and C-band synthetic aperture radar", International Journal of remote sensing, 25 Nov 2010

[Earth2012] Earth Observation Research Branch Team, Agriculture and Agri-Food Canada," Crop Identification and BBCH Staging Manual: SMAP-12 Field Campaign", https://smapvex12.espaceweb.usherbrooke.ca/BBCH_STAGI NG_MANUAL_CEREALS_CORN.pdf

[Lu96] S.-Y. Lu and R. A. Chipman, "Interpretation of Mueller matrices based on polar decomposition," J. Opt. Soc. Amer. A, Opt. Image Sci., vol. 13, no. 5, pp. 1106-1113, May 1996.

[McNairn2004] McNairn, H., and B. Brisco (2004), The application of C-band polarimetric SAR for agriculture: A review, Can. J. Remote Sens., 30(3).

[McNairn2014] McNairn, H., Wiseman, G., J. Powers, J., Merzouki, A., and Shang, J., "Assessment of Disease Risk in Canola using Multi-Frequency SAR: Preliminary Results ", to be published in EUSAR conference, 2014.

[Praks09] Praks, J: Colin-Koeniguer, E; Hallikainen, M. T, "Alternatives to target entropy and alpha angle in SAR Polarimetry", IEEE Trans. On Geosci. Remote Sensing, Vol. 1. 7, pp. 2262-2274, 2009. 
The International Archives of the Photogrammetry, Remote Sensing and Spatial Information Sciences, Volume XL-7/W3, 2015

36th International Symposium on Remote Sensing of Environment, 11-15 May 2015, Berlin, Germany

[SMAPVEX2012a] SMAPVEX 12 web site: https://smapvex12.espaceweb.usherbrooke.ca/home.php;

[SMAPVEX2012b] SMAPVEX12 experimental site SMAPVEX-CropMap_SMAPVEX_Area.rar,

https://smapvex12.espaceweb.usherbrooke.ca/intranet.php

[SMAPVEX12 Database Report]

https://smapvex12.espaceweb.usherbrooke.ca/SMAPVEX12_D

atabase_Report_final.pdf, December 18, 2012 